\title{
NUMERICAL SIMULATION OF AIR - WATER FLOWS IN AN EVAPORATIVE CONDENSER
}

\begin{abstract}
I. C. Acunha Jr a, and P. S. Schneider ${ }^{\mathrm{b}}$

${ }^{a}$ Instituto Federal de Educação Ciência e Tecnologia

do Rio Grande do Sul

Refrigeração e Climatização

Rua Alfredo Huch, 475

CEP 96.201-460, Rio Grande, Brasil

ivoni.acunha@riogrande.ifrs.edu.br

${ }^{\mathrm{b}}$ Universidade Federal do Rio Grande do Sul

Departamento de Engenharia Mecânica

Rua Sarmento Leite, 425

CEP 90.050-170, Porto Alegre, Brasil pss@mecanica.ufrgs.br

ABSTRACT

Evaporative condensers present a hard problem for numerical modeling because of the complex phenomena of heat and mass transfer outside of the bundle tubes in turbulent flows. The goal of this work is to study the air and water behavior inside an evaporative condenser operating with ammonia as the refrigerant fluid. A commercial CFD software package (FLUENT) is employed to predict the two-phase flow of air and water droplets in this equipment. The air flow is modeled as a continuous phase using the Eulerian approach while the droplets water flow is modeled as a disperse phase with Lagrangian approach. The coupling between pressure and velocity fields is performed by the SIMPLE algorithm. The pressure, velocity and temperature fields are used to perform qualitative analyses to identify functional aspects of the condenser, while the temperature and the relative humidity evolution contributed to verify the agreement between the results obtained with the numerical model and those presented by equipment manufacturer.
\end{abstract}

\section{NOMENCLATURE}

I unitary tensor

$C_{D} \quad$ drag coefficient

d diameter, $\mathrm{m}$

$D_{i_{\mathrm{r}} \mathrm{m}}$ mass diffusion coefficient of specie $i, \mathrm{~m}^{2} / \mathrm{s}$

E energy, $\mathrm{J}$

$F_{D}\left(v-v_{p}\right)$ drag force per unit particle mass, $\mathrm{m} / \mathrm{s}^{2}$

$\vec{F}$

momentum source, $\mathrm{kg} /\left(\mathrm{s}^{2} \mathrm{~m}^{3}\right)$

$\mathrm{g}$ gravity acceleration, $\mathrm{m} / \mathrm{s}^{2}$

$h$ enthalpy, $\mathrm{J} / \mathrm{kg}$

$\overrightarrow{\mathrm{I}}$ - diffusion flux of species, $\mathrm{kg} /\left(\mathrm{s} \mathrm{m}^{2}\right)$

$k$ thermal conductivity, $\mathrm{W} /\left(\mathrm{m}^{2}{ }^{\circ} \mathrm{C}\right)$

$p$ pressure, pa

Re Reynolds number

$R_{i} \quad$ rate of production of species $i$ by chemical reaction

$S_{C t} \quad$ effective Schmidt number for turbulent flow

$S_{i} \quad$ rate of creation by addition from the discrete phase

$S_{m} \quad$ mass source, $\mathrm{kg} / \mathrm{m}^{3}$

$S_{\boldsymbol{h}} \quad$ volumetric heat source, $\mathrm{W} / \mathrm{m}^{3}$

$\mathrm{t}$ time, $\mathrm{s}$

$\vec{v} \quad$ velocity, $\mathrm{m} / \mathrm{s}$

$\mathrm{Y}$ mass fraction

Greek symbols

$\rho \quad$ density, $\mathrm{kg} / \mathrm{m}^{3}$
Keywords: Numerical simulation, evaporative condenser, mass transfer, heat transfer, evaporative cooler modeling.

$\begin{array}{ll}\bar{\tau} & \text { tension tensor } \\ \mu_{t} & \text { molecular viscosity, } \mathrm{kg} /(\mathrm{s} \mathrm{m}) \\ \mu_{t} & \text { turbulent viscosity }\end{array}$

\section{Subscripts}

$i, j \quad$ species

$m$ mixture

p particle (droplet)

\section{INTRODUCTION}

The analysis of equipment related to the condensation has a key role in large-scale vapor compression refrigeration systems, especially those operating with ammonia as the refrigerant. This is because condensers with better effectiveness can promote systems with lower initial and operational costs.

The condensing water systems are among the main ones systems used in large-capacity systems where the advantages of lower consumption and stable operation overlap the disadvantages of keeping the condensers as treatment and restoration of water (Bueno,2006).

The logical development of the combination of a heat exchanger with a cooling tower is the evaporative condenser that uses spray water cooling process, characteristic of a cooling tower for heat transfer from process fluid in the heat exchanger (Donni, 2006). Thus, one can done an analogy between the phenomena that occurs in evaporative condensers and cooling towers, especially those operating in closed circuit which experiences the same functional structure that evaporative condensers 
(Fig. 1) having a fluid flows internally to the tubes (responsible for the rate heat transferred to the external environment) and externally, the spray water flowing in counterflow with the air. The identification of possible inefficiencies related to the flow of air or water can be revealed by computer simulation, making mathematical modeling and rendering application of CFD (computational fluid dynamics) important tools in this field.

Studies involving tests of evaporative condensers have shown good agreement between simulations and experimental measurements (Centeno et al., 2004, Centeno, 2005, Flores, 2005, Nakalski et al., 2006).

The analysis, through simulation of a largescale cooling tower (Krahe et al., 1998) showed the existence of problems due to the recirculation of air suction being above the exit at admission to the tower. This simulation could also serve to highlight the correlation between the mass flow of air and heating power dissipated by the tower.

The study carried out by Al-Waked and Behn (2007) presents the results of the application of CFD in a three-dimensional numerical simulation of the operation of a cooling natural draft. The CFD model has utilized the standard $k-\varepsilon$ turbulence model in the Fluent software through discrete phase model, where the air flow was solved as a continuous phase using the Eulerian approach. The water droplets trajectory was simulated as a dispersed phase, using the Lagrangian approach. The heat transfer from the water droplets in to surrounding air inside cooling tower consists of both convective and evaporative heat transfer, whereas heat transfer due to radiation is not significant, and has been neglected.

Another simulation of the cooling tower (Willianson et al., 2008) presents a two-dimensional and axisymmetric biphasic simulation of heat transfer and mass, where the water droplets in the spray and inside the cooling tower were represented with trajectories of drops also written in Lagrangian approach using a computational model in FLUENT package, that account the coupling of heat and mass transfer between droplets and air.

The model was developed to solve the problem in a steady state through the Reynolds Averaged Navier-Stokes Equations (RANS) using $k-\varepsilon$ standard turbulence model with buoyancy terms included in the transport equations of $k$ and $\varepsilon$. A semi-implicit method for the coupled velocitypressure fields was used through the method of discretization with second order upwind scheme for the advective terms.

The results of these simulations showed good agreement with data from experimental measurements.

The characterization and selection of the model being used to simulate the distribution of diameter and evaporation prove to be quite important in the work done revealing the influence of particle diameter and relative humidity on the phenomenon of evaporation.

Sun et al. (2007) studied a ventilated environment by using a CFD modified flow model, where the evaporation rate and the speed drag is expressed algebraically as a function of droplet diameter and also highlighting the importance of parameters such as the evaporation time and speed drag drops to the distribution of evaporating droplets. The evaporation rate is shown with an almost linear variation with the droplet diameter for diameters up to $80 \mu \mathrm{m}$, this relationship is no longer linear for droplet diameters greater than or equal to $100 \mu \mathrm{m}$. For particles with diameters less than or equal to $50 \mu \mathrm{m}$ could model the particles as an aerosol. The influence of relative humidity in the environment was also studied, showing a higher evaporation rate to lower relative humidities in the environment.

Gan et al. (2001) analyzed the performance of three cooling towers operating in closed circuit, using CFD as a tool. The computational model built considered the air as a continuous phase and water droplets as dispersed phase. The dispersed phase consisted of spherical drops of water and its influence on the continuo was developed through the coupling of heat, mass and momentum equations between the two phases.

The tubes were simulated as solid cylinders where heat transfer from tubes to the external environment was computed by two ways: as a volumetric heat generation (simulated for tower 1) and as constant heat flux uniformly distributed over the heat exchanger (simulated for tower 2). Simulating heat transfer as a term of volumetric heat generation, the tube temperature is found to increase slowly with iteration. Considering the heat flux prescribed constant and evenly distributed along the tube bundle, a good agreement between measured and simulated values was obtained with the operation of the tower at full load. With the cooling tower operating at lower flow better agreement between simulations and measurements was found using as boundary condition the heat flux linearly distributed along the tube bundle, assuming that the heat transfer in the first row of tubes is equal to twice the heat transfer that occurs in the latter. The distribution of air velocities in the tower without deflectors showed higher values in central and stagnation points in places near the wall. Due to higher air velocities in the central region at the bottom of the tube bundle drops tend to move toward the wall, decreasing the effectiveness of heat transfer. The problem of disposing of the drops was softened with the increase of their diameters, however the rate of evaporation in this region was reduced. The installation of deflectors between the output of the blower and tube bundle proved to be the best solution for distribution of air and evaporation.

The simulation of a hybrid cooling tower in closed circuit through commercial software 
(FLUENT) made by Sarker et al. (2008) also showed good agreement with experimental data. The standard $k-\varepsilon$ turbulence model is used, with the flow considered incompressible and steady state. The coupling between the fields of pressure and temperature was made by the algorithm SIMPLEC. The problem was solved in a two-dimensional, adiabatic simulation, with no heat transfer between the equipment and the surrounding. For further simplification, an area that includes half of the tower was used, due the symmetry problem. The tubes were represented by cylinders with internal heat generation.

The comparison of the performance of a prototype of a cooling tower in closed circuit from experimental measurements with those obtained through analysis and computer modeling done by Hasan and Sirén (2002) revealed the importance of parameters such as pitch tubes, number and rows of pipes, flow of air and water, air velocity and pressure loss in the ability to reject heat from the tower and the power absorbed by it in this way defining the tower coefficient of performance (COP) which was the objective of optimization. The prototype used was built to operate under conditions that result in nominal flow rates respectively equal to $3 \mathrm{~kg} / \mathrm{s}, 0.8$ $\mathrm{kg} / \mathrm{s}$ and $1.37 \mathrm{~kg} / \mathrm{s}$ for air, cooling water and spray water on the pipes. This work also defined the mass transfer coefficient for the case study. Since this parameter is strongly influenced by the Reynolds number, the correlation is a function of mass velocity of air and very good agreement with the results presented by the correlations used in the literature (parker and Treybal, 1961, Mizushina et al, 1967, Niitsu $^{a}$ et al. 1967, Niitsu ${ }^{b}$ et al., 1967, Niitsu et al., 1969).

The behavior of air, cooling water and the spray of water was also reported by Hasan and Sirén (2002), showing how these parameters evolve in a vertical center line in the cooling tower from the first contact until the last tube.

A model to study the evaporative cooling process that occurs in a new type of cooling tower, called Hidrosolar Roof was developed by Kaiser et al. (2005). This tower has a smaller fall of drops and the use of renewable energy in place of fans to provide the air flow inside the tower. The model uses the Eulerian approach for air and the Lagrangian approach for water droplets with the coupling between these phases done as follows: the algorithm computes the heat, mass and momentum exchanged between the air and the particles which follow trajectories. These values are incorporated in the subsequent continuous phase calculations. This problem was solved using Fluent software through a two-dimensional simulation, where the equations were discretized using the PRESTO scheme with UPWIND second-order scheme for the advective terms, with the coupling between pressure and velocity fields made through SIMPLE algorithms.
Properties of air such as specific humidity, enthalpy, and others were calculated using equations for psychrometric properties derived from fundamental equations for mixtures of ideal gases to assess the behavior of the efficiency of the tower considering the variation of parameters such as maximum temperature difference water cooling, the ratio between the flow of water and air, wet bulb temperature and the average diameter of the droplets, eventually establishing a numerical correlation that incorporates all these variations in the efficiency of the equipment studied. The validation of results was performed by comparison with experimental data.

The works cited above were the basis for constructing the model used in the simulation of evaporative condenser studied as these, for the most part, focus on the modeling of thermal systems by using discrete phase for the spray water, and $k-\varepsilon$ turbulence model. They also demonstrate the possibility of two-dimensional simulation and symmetry condition, and that several hypotheses are discussed for the boundary conditions of the pipes.

\section{COMPUTATIONAL MODEL}

This paper studied an evaporative condenser using CFD (Computational Fluid Dynamics). The commercial software FLUENT based on the finite volume method (FVM) was used to simulate air and water flows outside the tubes. The air, modeled as a continuous phase using the Eulerian approach, consists of a mixture of air and water vapor, while the water droplets were modeled as dispersed phase through the Lagrangian approach. The model was solved according to the implicit transient formulation. The Realizable $\mathrm{k}-\varepsilon$ turbulence was adopted. The effects of buoyancy and standard wall function were considered. The SIMPLE algorithm was used for the coupling of pressure and velocity fields. Because the length of the condenser is much larger than the height and width, the flow was considered two-dimensional. It was considered also adiabatic, with no heat transfer between the equipment and the surrounding. For further simplification, a field considering only half of the evaporative condenser was used, due to the symmetry presented by the problem.

\section{GOVERNING EQUATIONS FOR AIR}

The modeling for the turbulent flow of the continuous phase (air), including heat and mass transfer between this and the dispersed phase consists of conservation equations of mass, momentum energy and species. The mass conservation equation is written as follows:

$$
\frac{d p}{\partial t}+\mathbb{V}(\rho \vec{z})=S_{m}
$$


where $t$ is time (s) $\rho$ is the density $\left(\mathrm{kg} / \mathrm{m}^{3}\right), v$ is velocity $(\mathrm{m} / \mathrm{s})$ and $S_{m}$ is a source term that includes the addition of mass by the discrete phase due to vaporization of water droplets.

The following equations represent the conservation of momentum which includes the source term $(\overrightarrow{\mathbf{F}})$ due to interaction with the discrete phase and the tension tensor $(\mathbf{V}(\bar{T})$ ):

$$
\begin{gathered}
\frac{\partial}{\partial t}(\rho \vec{v})+\nabla(\rho \vec{v})=-\nabla p+\nabla(\vec{v})+\rho \vec{g}+\vec{k} \\
\bar{\tau}=\mu\left[\left(\nabla \vec{v}+\nabla v^{+}\right)-\frac{2}{3} \nabla \vec{v}\right]
\end{gathered}
$$

where $H$ is viscosity molecular $(\mathrm{kg} / \mathrm{ms})$ and $I$ is the unit tensor.

The energy is calculated as follows:

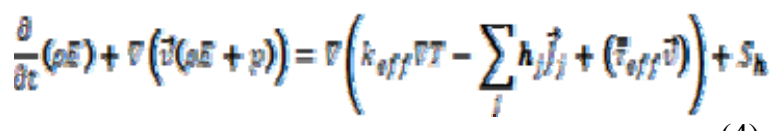

where $k_{\text {eff }}$ is the effective thermal conductivity $(\mathrm{W} /(\mathrm{mK}))$, corresponding to $k+k_{t}$ where $k_{t}$ corresponds to turbulent thermal conductivity, $\vec{J}_{\vec{i}}$ is the mass diffusion flux of species $j\left(\mathrm{~kg} / \mathrm{m}^{2} \mathrm{~s}\right)$. The first two terms on the right side of the equation refer to energy transferred by heat diffusion and mass diffusion, respectively. The third term corresponds to viscous dissipation, which was neglected. The energy $E$ shown in Eq. (4) is given by:

$$
E=h-\frac{p}{p}+\frac{v^{2}}{2}
$$

The species are conserved according to Eq. (6)

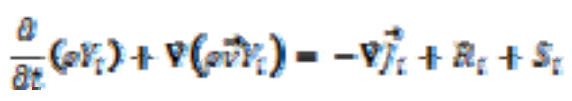

In this equation, $Y_{i}$ is mass fraction of each species $i$, $R_{i}$ is the production of species by chemical reaction and $S_{i}$ is the rate of creation by the addition of the dispersed phase. The production of species by chemical reaction was also neglected. The flow of mass diffusion equation used in the energy and species conservation equation is calculated as follows:

$$
\vec{h}_{t}=-\left(\rho D_{i, m}+\frac{\beta F_{\varepsilon}}{S_{C t}}\right) \nabla Y_{t}
$$

where $S_{C t}$ is the turbulent Schmidt number which is given by $\frac{\sigma_{t}}{D_{t}}, \mu_{z}$ being the turbulent viscosity and $D_{t}$ turbulent diffusivity.

\section{GOVERNING EQUATIONS FOR WATER}

The dispersed phase consists of spherical particles of water being injected at six points per injection plane. The trajectory of the discrete phase particles can be determined by making the integration of the balance of forces on particles using Lagrangian reference. This force balance equates the particle inertia forces with the forces acting on the particles [11] and can be written in the form of force per unit mass as follows:

$$
\frac{d v_{p}}{d t}=F_{z}\left(v-v_{p}\right)+\frac{g\left(\rho_{p}-\rho\right)}{\theta_{p}}+\frac{\rho}{p_{p}} v_{p} \frac{\partial v}{d n_{p}}+\frac{1}{2} \frac{\rho}{p_{p}} \frac{d}{d t}\left(v-v_{p}\right)
$$

where $r_{p}$ is the trajectory of each particle and, $v$ and $v_{p}$ are the velocities $(\mathrm{m} / \mathrm{s})$ of the continuous phase and particles, respectively. The second term on the right side of the equation represents the gravitational force per unit mass, and the third, also on the right side of this equation, represents an additional force per unit mass that occurs due to the pressure gradient in the fluid. The last term is due to acceleration caused in the fluid surrounding the particle, which is important in cases where $\rho>\rho_{p}$ [20]. The first term refers to the drag force per unit mass experienced by the particles which is given by:

$$
F_{D}=\frac{18 \xi}{P_{p} d^{2}} \frac{C_{D} R e}{24}
$$

where $d_{p}$ is the diameter (m) of the particles. The Reynolds number $(\mathrm{Re})$ is calculated by the relative velocity between particles and continuous phase, defined as:

$$
R \theta=\frac{\rho d_{p}\left|n_{p}-v\right|}{\alpha}
$$

The drag coefficient $C_{D}$ is calculated with the aid of the following equation. The constants $a_{1}, a_{2}$ and $a_{3}$ are constants given by Morsi and Alexander for spherical particles under different ranges of Reynolds numbers [20].

$$
C_{D}=a_{1}+\frac{a_{g}}{R e}+\frac{a_{g}}{R e^{2}}
$$




\section{BOUNDARY CONDITIONS}

The simulation of the condenser was made with the injection of water droplets through holes of $8 \mathrm{~mm}$ in diameter. The water is injected on small plates that promote the spreading of droplets on the tube bank. As the simulation done in 2D considers the unitary length, the flow of water and air used for simulation correspond to $1 / 6$ of the flow of air and water from the real condenser as it has $6 \mathrm{~m}$ in length. The condition of symmetry of the problem allowed simulation of only half of the geometry, thus establishing a vertical line of symmetry in the center of the condenser.

The spray water temperature is $302 \mathrm{~K}$, and is sprayed on the plates spread at a rate of $6 \mathrm{~kg} / \mathrm{s}$.

The air is admitted into the condenser under atmospheric pressure at an angle of $45^{\circ}$ with the horizontal toward the condenser water sump with temperature of $302.6 \mathrm{~K}$ and relative humidity of $45.3 \%$

At the air outlet a total manometric pressure of $370 \mathrm{~Pa}$ was considered at the ventilator intake, with temperature of $303.4 \mathrm{~K}$ and relative humidity of $90.7 \%$. These conditions ensure an air flow rate of approximately $7.4 \mathrm{~kg} / \mathrm{s}$.

In tubes bundles two different conditions were simulated: prescribed temperature and prescribed heat flux. The condition of prescribed temperature was realized and referred to as three zones with different temperatures: dessuperheating (temperature equal to $393 \mathrm{~K}$ ), condensation (temperature equal to $308 \mathrm{~K}$ ) and subcooling (temperature equal to $303 \mathrm{~K}$ ). The heat flux prescribed also took into consideration the three zones covering the three phenomena to different extents. Two situations were investigated regarding the behavior of the particles to impinge on the bank of tubes: the first was adopted as a boundary condition, reflection of the drops when they impact against the tubes, using coefficients of restitution for the normal and tangential moments values of 0.2 and 1.0 , respectively. The second possibility considers that droplets can become a film on the tubes in which each particle can stick, rebound, spread or splash.

\section{RESULTS AND DISCUSSION}

The pressure loss of air flow in an evaporative condenser is a factor of great importance because it directly influences the costs of manufacturing and operating such equipment. The intensity of the pressure drop is mainly due to factors such as slope of deflectors in the air inlet openings, tubes arrangement, tube spacing of the bank in relation to the walls, geometry and spacing of the drift eliminators and position of the ventilator in relation to the condenser. The speed (and therefore the flow) of air is strongly influenced by the resistance offered by the condenser air flow. Figure $2 \mathrm{a}$ shows the pressure field obtained from the simulation. The pressure in the condenser is reduced with the height of the same, being smaller the closer it is to the exhaust air, as expected. At the bottom of the tube bank there is no significant difference in pressure, except in the vortex that appears just above the air inlet. This vortex is due to the tilt in the direction of air flow to promote better air distribution in the rest of the condenser. When passing through the tube bank air suffers a slight pressure drop, which intensifies when passing by the drift eliminators. These also contribute to the formation of a nonhomogeneous distribution of air in the fan intake, providing a stream of air with significant changes of direction. This behavior can also be observed in the velocity field shown in Figure $2 b$ using the outlines formed by different speeds experienced from input to output. The highest speeds are at the top of the entrance (below the vortex) and sides of the tube bank, where there is a spacing that offers less flow resistance than the spacing between tubes, providing a preferential outlet area, but inert to the heat transferred directly by the tubes. Another feature that has no substantial benefits to the condenser is the upper corner where speeds tend to zero as the tubes are located just above the vortex. Since the advection of these is above zero, these pipes do not give significant effect on the transfer of heat to the condenser. Their no operation, however, is obscured by the fact that this is an area of possible subcooling. Above the drop spreaders it is possible to observe the influence of sprayed water, since the speed of the continuous phase tends to zero.

The variation of air temperature in the condenser due to the vertical at the center of the tube bank and center of the condenser is shown in the graphs of Figs. 3 and 4.

These graphs represented in green lines symbolize the area where the air is within the condenser. In Figure 3 the temperature variation due to the vertical position is shown for the condition of heat flux prescribed on the tubes bank. In the tray just below the air inlet, there is a temperature gradient showing that the temperatures are lower, as the lower region of the condenser gets closer. In the entrance region the temperature variation is slight, with a greater slope next to the tubes. 


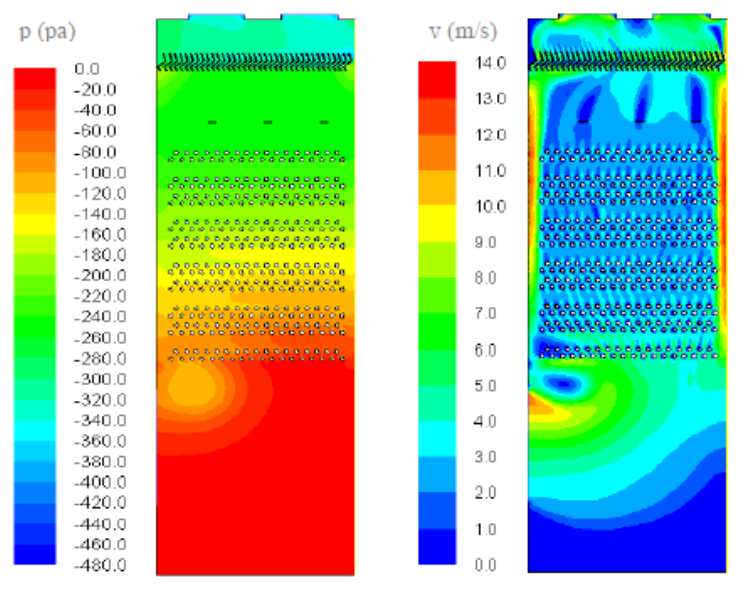

Figure 2. a) pressure field, b) Velocity field.

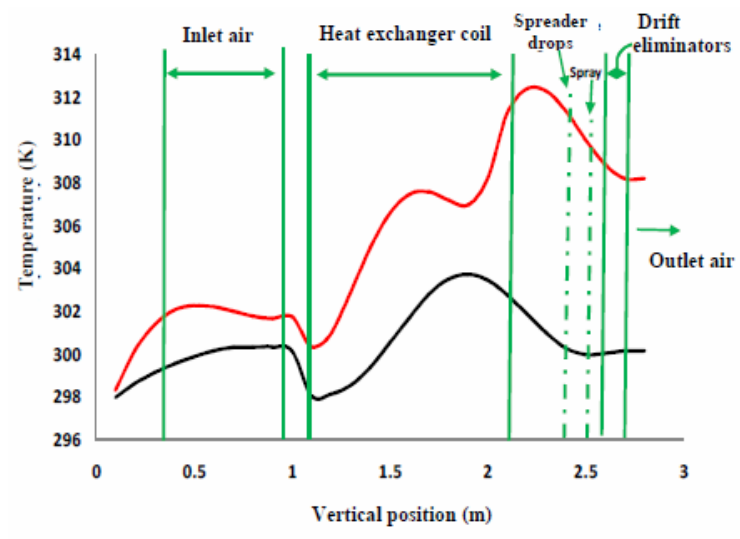

Figure 3. Profile of air temperature at the center of the condenser (-) and in the center of the tubes bank $(-)$, for the condition of prescribed heat flux in the bundle tube.

When passing by the bank of tubes the air temperature and moisture content are increased, reaching the maximum temperature as it leaves the last row of tubes. From this point a further reduction in temperature begins due to the increase of moisture content without the transfer of heat to the refrigerant. In the center of the condenser (shown in black in the graph) a similar trend occurs, however, the peak temperature just before the scattering region drops. This trend probably occurs due to the center of the condenser not being submitted directly to pipes heat, especially the region of dessuperheating. Moreover, near this region there are the higher speeds, making the phenomenon advection more intense.

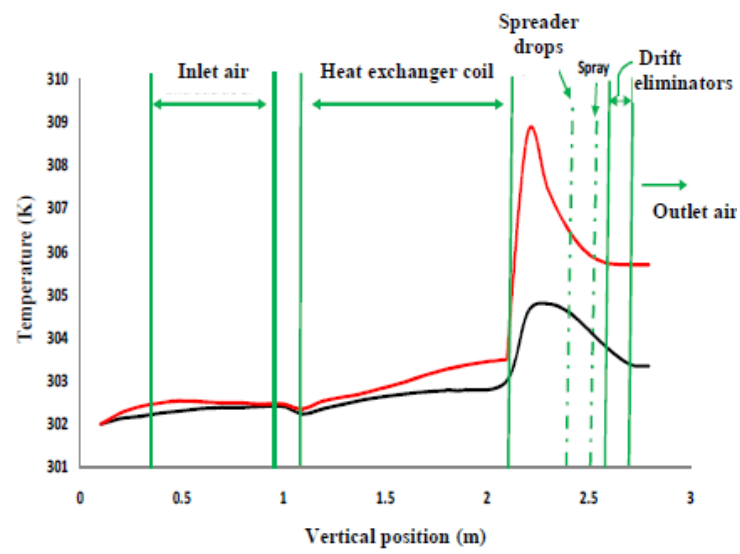

Figure 4. Profile of temperature at the center of the condenser (-) and in the center of the tube bank (-), for the condition of prescribed temperature in the tube bundle.

The behavior shown in Figure 4 shows a temperature rise much more on dessuperheating zone where the temperature is prescribed at $393 \mathrm{~K}$ (corresponding to the compressor discharge temperature observed for the compression ratio in which it operated during the tests). With this boundary condition, a minor variation also occurs in the center of the condenser in the center of the tube bank.

\section{CONCLUSIONS}

The study presented in this paper shows some results obtained by numerical simulation of an evaporative condenser with a capacity of approximately $1700 \mathrm{~kW}$ operating at a condensing temperature of the refrigerant (R-717) of $308 \mathrm{~K}$.

Some characteristics of the flow with respect to pressure and velocity were observed, and some areas that could be improved by changing the geometry identified. The use of deflectors with different angles along the air inlet may attenuate the effects caused by vortex in the entrance region, and hence improve the heat transfer in tubes located immediately above this.

The study of new geometries for the drift eliminators, along the scattering characteristics of droplets and sprays, may reduce the air flow pressure drop, increasing the coefficient of performance of the condenser. Having seen the importance of evaporation in the process and this being the area that provides greater wetting, an increase in vertical dimension between the condenser tube bank and the drift eliminators can provide a more effective cooling of water before reaching the tubes.

When considering the formation of a film when the particles collide with the tubes, a worse controllability with respect to the number of particles formed was obtained in addition to causing the evaporation from the surface of the pipes that it is inappropriate for this application. 
The results that were obtained for the condition of prescribed heat flux in tubes showed higher temperatures than the temperatures that occur inside the condenser in operation, because in most of part of the tubes bank region this temperature is limited to condensing temperature. The condition of prescribed temperature is shown as a best choice among those studied. To improve the approximation to the actual condition of operation, the use of the coefficient of heat transfer tubes as the internal boundary condition is being studied, where its value is used in each row of the tube bank, taking into account the dessuperheating area, the title and area of subcooling.

\section{ACKNOWLEDGEMENTS}

The first author thanks the Frost Frio Refrigeração Industrial company by providing data and equipment and $\mathrm{CNPq}$ by his doctorate scholarship that also contributed significantly to this work.

\section{REFERENCES}

Al-Waked Rafat, and Behnia M., 2007, Enhancing Performance of Wet Cooling Towers, Energy Conversion \& Management, Vol.48 pp. 2638-2648.

Bueno, O. S., 2006, Resfriamento Sensível da Água de Resfriamento. Congresso da indústria de climatização e refrigeração-Mercofrio, Porto Alegre.

Centeno, F. R., 2005, Análise teórico experimental do desempenho de condensadores evaporativos, Monografia, Universidade Federal do Rio Grande do Sul.

Centeno, F. R., França, F. H. R., Gomes, H. M., and Schneider, P. S., 2004, Aplicação de Redes Neurais Artificiais para Modelagem do Comportamento Térmico de Condensadores Evaporativos, 10th Brazilian Congress of Thermal Sciences and Engineering.

Donni, R. G., 2006, Estudo Comparativo Entre Trocadores de Calor Convencionais e Trocadores Evaporativos. Feira e congresso da indústria de climatização e refrigeração Mercofrio, Porto Alegre.

Flores F. S., 2005, Modelamento matemático de condensadores evaporativos pelo método $\varepsilon$-NUT, Monografia, Universidade Federal do Rio Grande do Sul.

FLUENT: User Guide, Fluent Inc., 2006. $<$ http://www.fluent.com>

Gan, G., Riffat, S. B., Shao, L., and Doherty, P., 2001, Application of CFD to closed-wet cooling towers, Applied Thermal Engineering, Vol. 21: pp. 79-92.
Hasan, A., and Sirén, K., 2002, Theoretical and computational analysis of closed wet cooling wet cooling towers and its applications in cooling of buildings, Energy and Buildings, Vol. 34, pp. 477486.

Kaiser, A. S., Lucas, M., Viedma, A., and Zamora, B., 2005, Numerical model of evaporative cooling processes in a new type of cooling tower, International Journal of Heat and Mass Transfer, Vol. 48, pp. 986 - 999

Krahe, R., França, and F. H. R. Vielmo, H. A., 1998, Análise Numérica de Uma Torre de Resfriamento de Grande Porte. Congresso da indústria de climatização e refrigeração Mercofrio, Porto Alegre.

Nakalski, A. T., Danieli, R., Schneider, P. S., and Beyer, P. O., 2006 Modelagem matemática do comportamento de condensadores evaporativos: Comparação entre duas abordagens, Congresso da indústria de climatização e refrigeração Mercofrio, Porto Alegre.

ParKer R. O., and Treybal, R. E., 1961, The heat mass transfer characteristics of evaporative coolers, Chemical Engineering Progress Symposium Series, Vol. 57, No. 32.

Sarker, M. M. A., Kim, E., Moon, C. G., and Yon J. I., 2008, Performance characteristics of the hybrid closed cooling tower, Energy and Buildings, Vol. 40, pp. 1529 - 1535.

Sun, W., Ji, J., Li, Y., and Xie, X., 2007, Dispersion and settling characteristics of evaporating droplets in ventilated room, Building and Environment, Vol. 42, pp. 1011-1017.

Mizushina, T., Ito, R., and Miyashita, H., 1967, Experimental study of an evaporative cooler, International Chemical Engineering Vol.7, No 4, pp. $727-732$.

Williamson, N., Armfield S., and Behnia, M., 2008, Numerical Simulation of Flow in a Natural Draft Wet Cooling Tower - The Effect of Radial Thermofluid Fields, Applied Thermal Engineering, Vol. 28, pp. 178-189.

Niitsua, Y., Naito, K., and Anzai, T., 1967, Studies on characteristics and design procedure of evaporative coolers, Journal of SHASE, Japan, Vol. 41, No. 12.

Niitsu ${ }^{b}$, Y., Naito, K. and Anzai, T., 1967, Studies on characteristics and design procedure of evaporative coolers, Journal of SHASE, Japan, Vol. 41, No. 13.

Niitsu, Y., Naito, K., and Anzai, T., 1969, Studies on characteristics and design procedure of evaporative coolers, Journal of SHASE, Japan, Vol. 43, No. 12.

Received: September 04, 2008

Revised: October 04, 2008

Accepted: November 04, 2008 